\title{
The dynamics of surge in compression systems
}

\author{
A N VISHWANATHA RAO ${ }^{1}$ and O N RAMESH ${ }^{2}$ \\ ${ }^{1}$ Gas Turbine Research Establishment, Bangalore \\ ${ }^{2}$ Department of Aerospace Engineering, Indian Institute of Science, \\ Bangalore 560012 \\ e-mail: anv_rao@yahoo.co.in; onr@aero.iisc.ernet.in
}

\begin{abstract}
In air-compression systems, instabilities occur during operation close to their peak pressure-rise capability. However, the peak efficiency of a compression system lies close to this region of instability. A surge is a violent mode of instability where there is total breakdown of flow in the system and pressure-rise capability is lost drastically. Generally, all compression systems operate with a margin defined as the 'surge margin', and, consequently, system operational efficiency is lower. It is of interest to study compression-system surge to understand its dynamics in order to operate compression systems close to the instability for achieving high efficiency safely without encountering surge. Unsteady pressure data from a compression system, captured during surge oscillations, reveal many aspects of flow physics and are analysed to understand the surge dynamics of the system. A set of controlled experiments was conducted with a simple desktop experimental test set-up and essential aspects of surge dynamics have been characterised.
\end{abstract}

Keywords. Compression system; surge; desktop experiments.

\section{Introduction}

Stable operation of engines is of primary interest in aircraft. Compressor stability plays a major role in overall engine stability. When an engine operates close to its peak performance point, flow within the compressor becomes unstable. Handling these instabilities is a great challenge to engine designers, since in some cases engines have to be shut off and restarted in order to get back to a stable operation.

Surges are global, self-excited instabilities that can affect the whole system and should be avoided. The occurrence of surge depends on the pressure ratio-mass flow characteristic of the compression system. It is characterised by large amplitude limit-cycle oscillations in mass flow rate and pressure ratio. Surge starts to occur in a region of the compressor map, where the pressure ratio/mass flow characteristics for a constant speed have a positive slope. In a compression system mechanical energy input into the system, is transformed to pressure energy of the fluid by a complex mechanical arrangement of rotating and stationary parts. The pressurised fluid, delivered by the compression system is subsequently stored in a plenum of large volume. Pressurising mechanism must always develop sufficient pressure, which in 
turn must maintain the fluid pressure in the plenum at the desired value. A sudden loss of pressurising capability of the system results in the stored fluid in the plenum discharging through the pressurising system itself. Such flow instability is termed as a surge.

Loss of pressurising capability of the compressor is the root cause of surge. In the case of aero-engine surge, it could be due to many reasons, either internal or external to the engine. Internal problems like compressor going into rotating stall, resulting in loss of pressurising capability, or external effects like inlet distortion, hot gas ingestion or even a bird strike, could lead to an engine surge.

Since, surge is a system response, surge models must consider all the components of the system Grietzer's (1976) model and its variants have addressed the problems of rotating stall and surge together. Grietzer (1976) considered the entire system while forming the governing equations. He arrived at a $B$ parameter $\left(=\frac{U_{t}}{2 \omega_{H} L_{c}}\right.$, where $U_{t}$ is the tip speed of the compressor blade, $\omega_{H}$ is the Helmholtz frequency and $L_{c}$ is the length of the duct), which was found to bifurcate the boundary between rotating stall and surge. However, the usage of the $B$ parameter is somewhat ad-hoc since it has no parameters of the compressor that decide the stability of the system. Even though the experimental work of Grietzer (1976) was quite in conformity with his theory, the parameter $B$, could not correctly predict surge and stall behaviour in many engines as reported by Cumpsty (1989).

It was reported by Day (1994) that during experimental work on stall and surge, oscillations at the system natural frequency existed in some cases without the system entering into surge cycles. Explanations for such system behaviour, which cannot be answered by B parameter alone, need to be developed.

It is also observed in many experimental and theoretical studies that surge oscillations are sinusoidal when the surge is mild and become increasingly non-sinusoidal (a nonlinear system behaviour) as it develops into a deep surge. In other words, deep surge cycles are similar to relaxation oscillations exhibited by a nonlinear oscillator. It is interesting to note that after the classical works of Emmons et al (1995), Grietzer (1976), Stenning (1980) etc., not many studies on theoretical modelling of compression system stability have been reported in the literature.

From the foregoing, it is clear that it is profitable to isolate surge phenomenon without including stall dynamics, since rotating stall is a local instability of the compressor, whereas a surge is a global instability phenomenon. In the present work, the focus is on rationally characterising the surges in the system, without getting into the details of the original cause of the surge, i.e. rotating stall, inlet distortion etc. A simple desktop model of a compression system that can exhibit essential features of a surge is attempted in order to characterise mild, classic, and deep surges.

\section{Experimental setup}

The desktop experimental setup is shown in figure 1. It consists of a centrifugal blower, a cylindrical duct, a diffuser, a plenum built with Perspex and a throttle valve. A variable speed centrifugal blower of capacity $0.025 \mathrm{~kg} / \mathrm{s}$ mass flow, controlled by a variable AC supply was used. The compressor duct was made long enough to contain the necessary instrumentation. A rectangular diffuser with internal fins was used to recover maximum possible static pressure from the flow. A plenum of volume $0.1296 \mathrm{~m}^{3}$ was used as the mass storage element. A throttle valve was used to control exhaust mass flow rate from plenum at any required flow rate.

Wall static-pressure measurements were made at several locations. Conventional surface static-pressure tappings were made with $0.5 \mathrm{~mm}$ diameter holes. Static pressure was sensed 


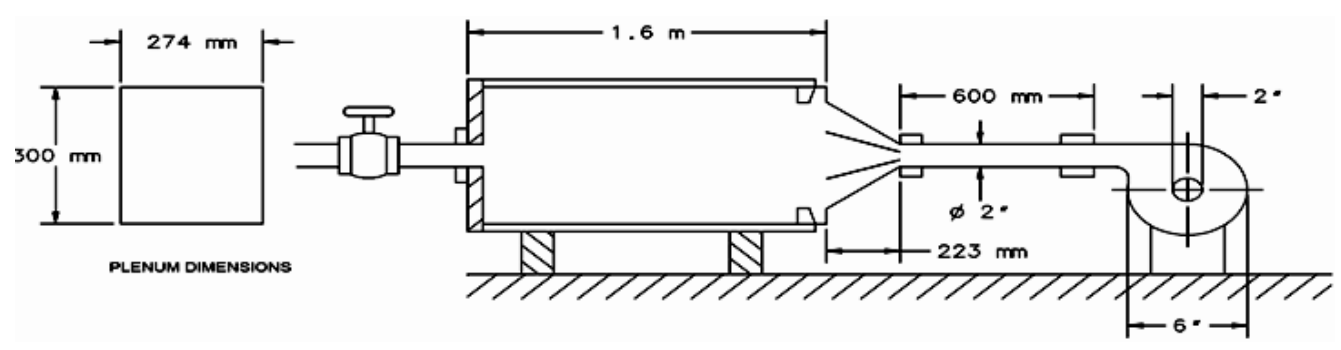

Figure 1. Experimental setup.

at three locations on the plenum casing, two locations on the compressor duct, and two locations on throttle duct. A few condenser microphones were mounted flush to the plenum wall, compressor delivery duct and impeller lead duct, which had a bandwidth of about $5 \mathrm{kHz}$. These condenser microphones were able to pickup pressure-blade pass frequency, stall frequencies and surge frequencies. There were no acoustic measurements made with these sensors. A pitot probe to sense flow velocity with an accurate differential pressure transmitter $(\mathrm{M} / \mathrm{S}$ Sentra, USA make, series $239 \mathrm{C})$ of range $+/-2.5$ inches $(1 \mathrm{inch}=2.54 \mathrm{~cm})$ of water column, was used for mass-flow measurement at the impeller entry. Measurement of plenum wall static-pressure gave the extent of pressure-rise of the system and hence another accurate measurement of this pressure signal was made with a pressure transmitter of range 15 inches of water column. In order to get fast response, sufficient bandwidth up to $400 \mathrm{~Hz}$, very short length pneumatic tubing (less than $10 \mathrm{~cm}, 3 \mathrm{~mm}$ diameter pipe) was used between measurement point and transmitter. Since the experiments were focused on surge signals which were less than $30 \mathrm{~Hz}$, values of damping in the pressure pulsations were very small.

Sensor output from pressure transmitters as well as condenser microphones had to be conditioned with simple operational amplifier based circuits in order to feed them to a computerbased data acquisition system. Pressure transmitter outputs were converted to DC voltage with a precision metal film resistor of $100 \Omega$, to get DC voltage in the range 0.4 to $1.2 \mathrm{~V}$.

For obtaining the characteristics of the pumping system, the blower was run at three speeds: 46 and 40 rps 60 (revolutions per second) and the throttle was progressively closed from fully open to fully close. Mass flow rate and pressure-rise across the blower were monitored and recorded. The characteristics are shown in figure 2. It can be seen that the mass vs. pressure-rise curves have negative slopes when mass is sufficiently high, and become almost zero-sloped curves when mass flow rates approach near zero values. As the peak pressurerise for the set speed approached, flow oscillations were observed. Hence, further readings could not be recorded. It can be seen that these curves are similar to typical radial compressor characteristics of a centrifugal compressor.

Surge oscillations were obtained, by running the blower at various speeds in the range from 20 to 60 rps. Surge oscillations were sinusoidal in most settings. Unsteady pressure signals were captured from condenser microphones and pressure transmitters, monitored online. These signals indicated limit-cycle surge oscillations around the system's natural frequency. A study was made to see whether surge oscillations were influenced either by blower speed or throttle settings (or in other words, the dependence on pressure ratio). It was found that the system's natural frequency was independent of either throttle setting or blower speed, i.e. the pressure ratio. However, the magnitude of surge cycles increased with higher pressure ratio. 


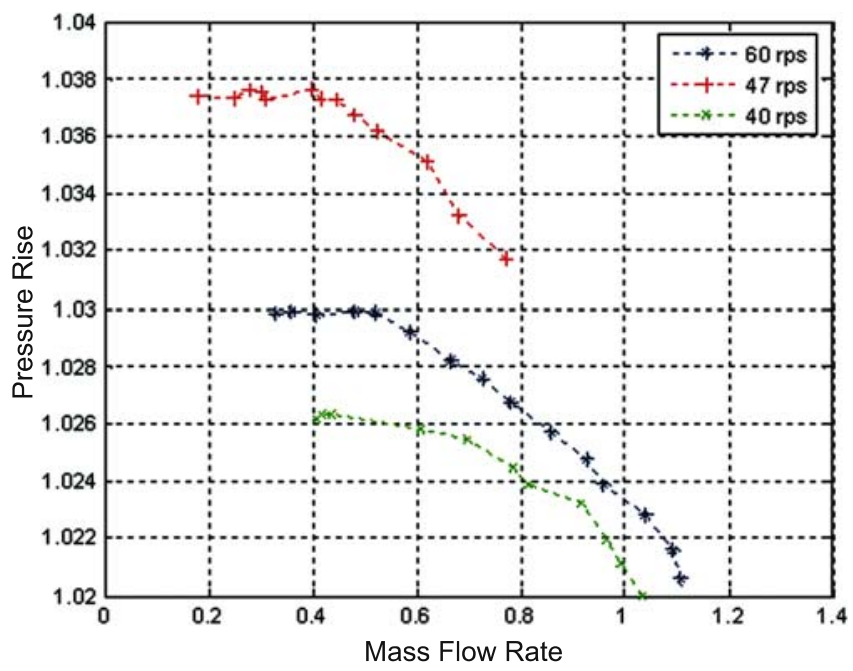

Figure 2. Centrifugal blower characteristics.

\section{Results}

Figure 3 shows the surge limit cycles, captured online on compressor characteristic plot. The horizontal axis is proportional to the mass flow rate and the vertical axis is the plenum pressurerise (in kilo pascals) above the ambient. It can be seen that there is reverse flow for part of the surge cycle. The time trace plots shown in figure $4 \mathrm{a}$ indicate sinusoidal oscillations in mass flow rate and plenum pressure. Figure $4 \mathrm{~b}$ illustrates the corresponding frequency spectra.

A parametric study of surge dynamics with changes in the system parameters was carried out. The initial setup did not have variability in plenum volume. After preliminary studies

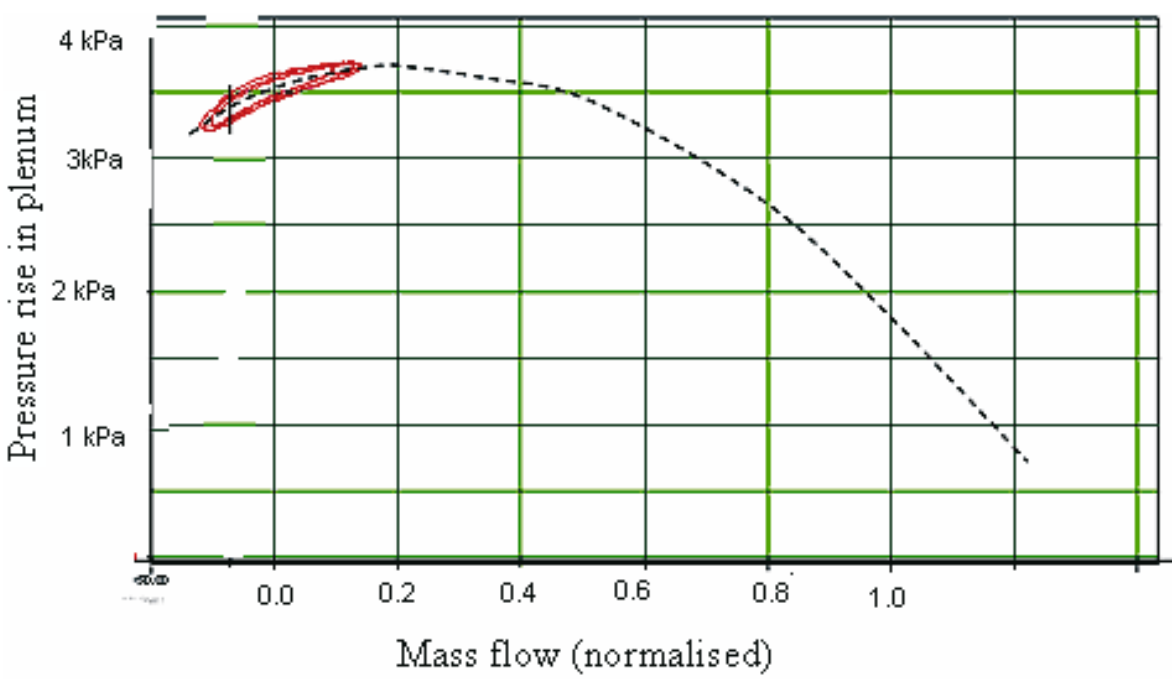

Figure 3. Online plot of surge cycles on a compressor characteristic. 


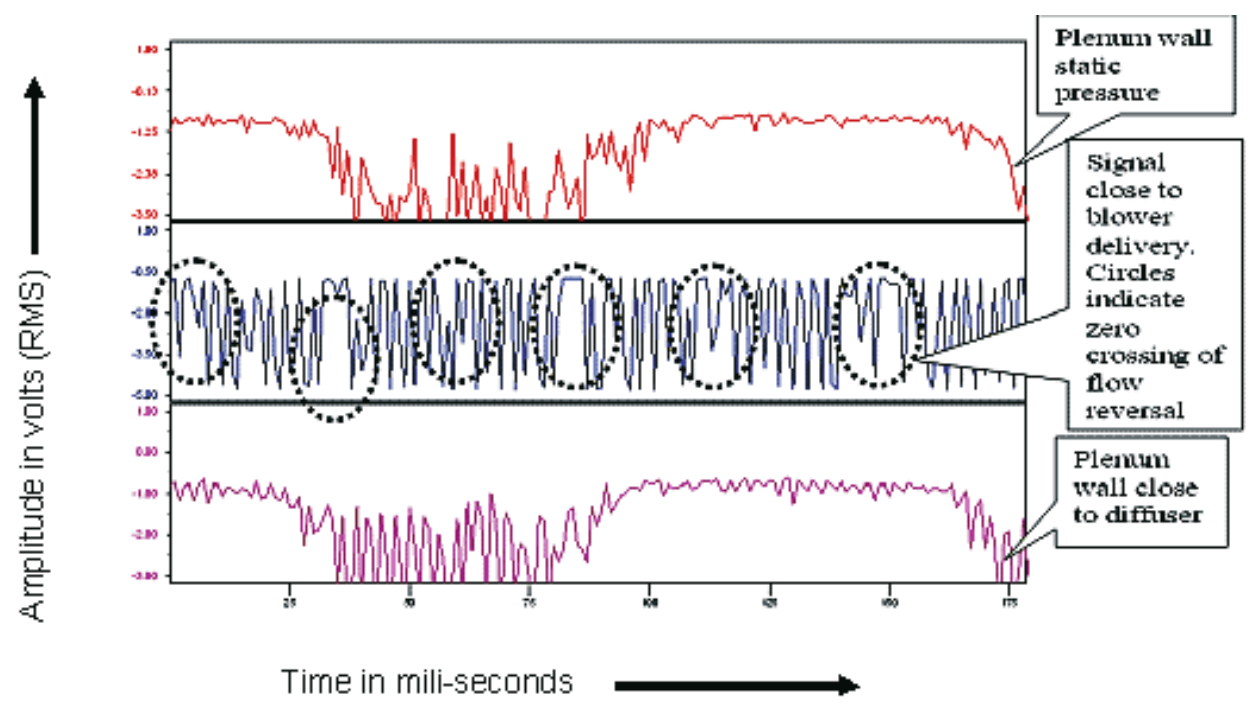

(a)

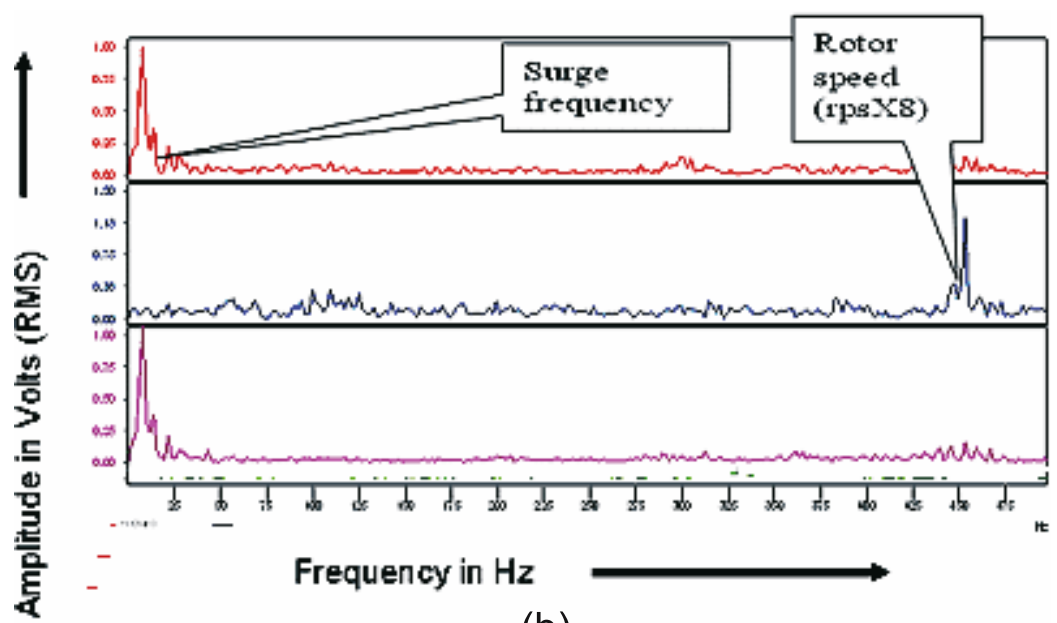

(b)

Figure 4. Time series (a) and corresponding frequency spectra plot (b) indicating surge.

of surge, flow visualisation studies, another setup was fabricated with a piston, cylinder configuration, with which volume of the plenum could be changed continuously.

Different lengths of compressor duct were used in the study. Tests were conducted with 1000,600 and $300 \mathrm{~mm}$ duct lengths.

Surge can be classified as mild, classic and deep. For mild and classic surges, there is no flow reversal. However, periodic pressure ratio fluctuations are small for mild surges whereas they are larger for classic surges. A mild surge is essentially a Helmholtz oscillator exhibiting sinusoidal oscillations. Such oscillations were observed in our setup with plenum volume set at $0 \cdot 129 \mathrm{~m}^{3}$, duct length set to $600 \mathrm{~mm}$ and by running the compressor at $45-50 \mathrm{rps}$ speed 


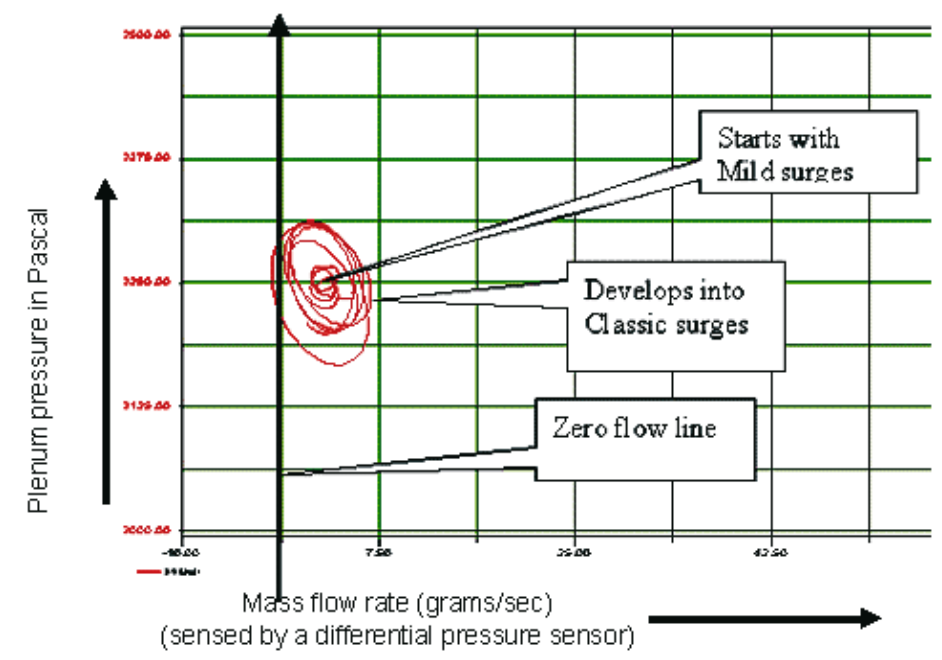

Figure 5. Classic and mild surges observed in the tests conducted. Abscissa shows mass flow rate and the ordinate shows the pressure-rise.

range. A compressor map was plotted online as shown in figure 5, which indicated mild surges as small inner circles, occasionally developing into classic surges.

Figure 6 shows the variation of measured surge frequency with the volume of the plenum. Greitzer's (1976) theory finds that the surge frequency is the same as the system natural frequency of the plenum (which acts like a Helmholtz resonator). However, this theoretical expectation is always about $20 \%$ higher than the measured frequency. This trend is also consistent with the experiments of Day (1994). This seems to be due to the isentropic assumption in the plenum in Greitzer's model which is not true in practice, where the rapidly varying flow pattern is irreversible.

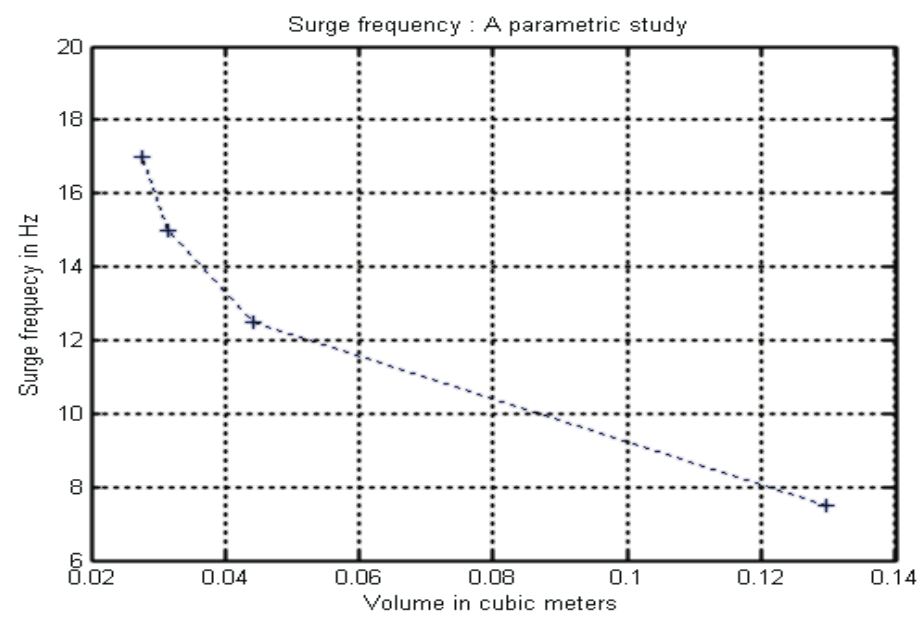

Figure 6. Variation of measured surge frequency with plenum volume. 


\section{Conclusions}

An inexpensive simple desktop experimental setup of a compression system, which exhibits surge oscillations has been realized. Mild and classic surges have been successfully produced on the desktop setup. This is for the first time that such a systematic study has been made in a desktop model and we believe this has great implications for the study of surges. The essential thermodynamic process in the experimental setup seems to be non-isentropic; classical theories over-predict the surge frequency as they involve isentropic assumptions.

It is with great pleasure that we dedicate this paper to Dr P R Viswanath on the occasion of his 60th birthday.

\section{References}

Cumpsty N 1989 Compressor aerodynamics (New York: John Wiley \& Sons)

Day I 1994 Axial compressor performance during surge. J. Propulsion Power, 10: 329-336

Emmons H, Paerson C, Grant H 1955 Compressor surge and stall propagation. ASME Trans. 77: 455-469

Greitzer E 1976 Surge and rotating stall in axial flow compressors. Part I: Theoretical compression system model. ASME J. Eng. Power 98: 191-198

Stenning A 1980 Rotating stall and surge. ASME J. Fluids Eng. 102: 14-20 